\title{
On the (In)Security of IDEA in Various Hashing Modes ${ }^{\star}$
}

\author{
Lei $\mathrm{Wei}^{1}$, Thomas Peyrin ${ }^{1}$, Przemysław Sokołowski ${ }^{2}$, \\ San Ling ${ }^{1}$, Josef Pieprzyk ${ }^{2}$, and Huaxiong Wang $^{1}$ \\ 1 Division of Mathematical Sciences, School of Physical and Mathematical Sciences, \\ Nanyang Technological University, Singapore \\ wl@pmail.ntu.edu.sg, thomas.peyrin@gmail.com \\ 2 Macquarie University, Australia
}

\begin{abstract}
In this article, we study the security of the IDEA block cipher when it is used in various simple-length or double-length hashing modes. Even though this cipher is still considered as secure, we show that one should avoid its use as internal primitive for block cipher based hashing. In particular, we are able to generate instantaneously free-start collisions for most modes, and even semi-free-start collisions, pseudo-preimages or hash collisions in practical complexity. This work shows a practical example of the gap that exists between secret-key and known or chosenkey security for block ciphers. Moreover, we also settle the 20-year-old standing open question concerning the security of the Abreast-DM and Tandem-DM double-length compression functions, originally invented to be instantiated with IDEA. Our attacks have been verified experimentally and work even for strengthened versions of IDEA with any number of rounds.
\end{abstract}

Keywords: IDEA, block cipher, hash function, cryptanalysis, collision, preimage.

\section{Introduction}

Hash functions are considered as a very important building block for many security and cryptography applications. Informally, a hash function $H$ is a function that takes an arbitrarily long message as input and outputs a fixed-length hash value of size $n$ bits. In cryptography, we want these functions to fulfill three security requirements, namely collision resistance and (second)-preimage resistance. It should be impossible for an adversary to find a collision (two different messages that lead to the same hash value) in less than $2^{n / 2}$ hash computations, or a

\footnotetext{
* The first, fourth and sixth authors are supported by the Singapore National Research Foundation under Research Grant NRF-CRP2-2007-03 and the first author is also supported by the Singapore Ministry of Education under Research Grant T206B2204 and by the NTU NAP Startup Grant M58110000. The second author is supported by the Lee Kuan Yew Postdoctoral Fellowship 2011 and the Singapore National Research Foundation Fellowship 2012.
}

A. Canteaut (Ed.): FSE 2012, LNCS 7549, pp. 163-179, 2012.

(C) International Association for Cryptologic Research 2012 
(second)-preimage (a message hashing to a given challenge) in less than $2^{n}$ hash computations. Most of nowadays hash functions divide the whole input message into blocks after padding it, and then process the blocks in an iterative way. A very known and utilised example is the Merkle-Damgaård algorithm [12 33], which uses an $n$-bit compression function $h$ in order to process the $m$ message blocks $M_{i}: C V_{i+1}=h\left(C V_{i}, M_{i}\right)$, where $C V_{i}$ is the $n$-bit internal state (or chaining variable) that is initialized by a fixed public value $C V_{0}=I V$ and the final hash value is $H_{m}$. This algorithm is very interesting because it allows to reduce the collision/preimage security of the hash function to the collision/preimage security of the compression function. However, in order to guarantee the soundness of the construction, a designer must ensure that an attacker can not break the collision/preimage resistance of the compression function. One can identify different security properties for a compression function:

- free-start collision: in less than $2^{n / 2}$ computations, find two different pairs $(C V, M) \neq\left(C V^{\prime}, M^{\prime}\right)$ such that they lead to the same compression function output value: $h(C V, M)=h\left(C V^{\prime}, M^{\prime}\right)$,

- semi-free-start collision: in less than $2^{n / 2}$ computations, find one chaining variable $C V$ and two different message blocks $M \neq M^{\prime}$ such that they lead to the same compression function output value: $h(C V, M)=h\left(C V, M^{\prime}\right)$,

- preimage: in less than $2^{n}$ computations, find one chaining variable $C V$ and one message block $M$ such that they lead to a given output challenge $X$ : $h(C V, M)=X$.

Note that a semi-free-start collision for the compression function where the chaining variable $C V$ is not chosen by the attacker directly leads to a collision for the whole hash function. In any case, a semi-free-start collision is very dangerous since it means that for some choices of $I V$, the attacker knows how to generate a collision. Even free-start collision are considered serious as they invalidate the collision resistance assumption on the compression function and we have seen many free-start collision attacks eventually turning into full hash collision attacks in the recent history (for example free-start collision attacks for MD5 were quickly identified [14, then upgraded to semi-free-start collision attacks [15] and eventually to full collision attacks [38]). As for preimage attacks on the compression function (also known as pseudo-preimages), they are very relevant since there exist a meet-in-the-middle algorithm that in most cases can turn them into a preimage attack for the full hash function.

The separation between a block cipher and a compression function has always been blurry. Constructions are known to turn the former into the latter [736] or the latter into the former [31. For example, the Davies-Meyer mode 1] converts a secure block cipher $E$ into a secure compression function and is incorporated in a large majority of the currently known hash functions. While very satisfying solutions exist to transform a secure $n$-bit block cipher into an $n$-bit compression function (Davies-Meyer, Miyaguchi-Preneel, Matyas-Meyer-Oseas modes [1] or see [736] for a systematic study of this problem), there is still a lot of research being actively conducted on double-block length compression functions (where 
the block cipher size is $n$ bits and the compression function output size is $2 n$ ), from simple-key block ciphers such as AES-128 or double-key such as AES-256 [11.

A major difference between the cryptanalysis of block ciphers and compression functions is that the attacker can fully control the inner behavior of the compression function. In other words, the attacker can use more efficiently the freedom degrees available on the input (i.e. the number of independent binary variables he has to determine). A new security model for block ciphers, the so-called known-key model 24, was recently proposed in order to fill the gap between these two situations. In this model, the secret key is known to the adversary and its goal is to distinguish the behavior of a random instance of the block cipher from the one of a random permutation by constructing a set of (plaintext, ciphertext) pairs satisfying an evasive property. Such a property is easy to check but impossible to achieve with the same complexity and a non-negligible probability using oracle accesses to a random permutation and its inverse. In general, these known-key attacks are not regarded as problematic when the block cipher is used in a classical "secret key" setting. Moreover, it is rare that such threats are extended to attacks on the compression function.

A potential candidate for hashing is the 64-bit block cipher IDEA [26 39] that uses 128-bit keys. While a simple-length hashing mode would only provide a 64-bit hash output, insufficient for most of nowadays security applications, a double-block length construction (DBL) would allow 128-bit hash outputs which can be sufficient in some scenarios. As IDEA handles double-length keys, more freedom in the constructions is possible. In fact, the well known Abreast-DM and Tandem-DM modes were specifically created to perform hashing with IDEA (see page 2 and Section 6 of [39]). These modes were later studied in much details [16 17/28/30], but the security they provide when instantiated with IDEA remains a 20-year-old standing open question. In classical "secret key" setting, IDEA has already been studied a lot [2/345|6|9|10/3 18] and is still considered as a secure cipher despite its age and despite the current best attack [5] that requires $2^{63}$ data (half the codebook) and $2^{114}$ computations to recover the secret key for IDEA reduced to 7.5 rounds over a total of 8.5 (the attack on the full cipher from [5] is very marginal with $2^{126.8}$ computations and the one from [22] requires $2^{126}$ computations and $2^{52}$ chosen plaintexts). One can also cite the work of [6], that exposes a weak-key class of size $2^{64}$. Note also that a first step towards analysis of IDEA in hashing mode was done in [21] where a 3-round chosen-key attack is described and in [9] where the authors show how to find a free-start near collision (only a subset of the output collides) when IDEA is plugged into the Hirose DBL mode 9] (and also a free-start collision if the internal constant $c$ is controlled by the attacker).

Our Contribution. In this paper, we study the security of the IDEA block cipher [26]39] when plugged into various block cipher based compression function constructions, such as the classical Davies-Meyer mode [1], also DBL constructions such as Hirose 1920, Abreast-DM and Tandem-DM 2739, Peyrin et al. (II) 35] or MJH-Double 29]. Even if this cipher is still considered as secure in the classical "secret key" setting, its security remains an open problem in hashing 
mode. Depending on the IDEA-based hash construction, we show that an attacker can find free-start collisions instantaneously, preimages or semi-free-start collisions practically. For some modes, we even describe a method to compute collisions for the whole hash function. These attacks are based on weak-keys utilisation, but in contrary to the "secret key" setting where the goal of the attacker is to exhibit the biggest weak-key class possible, in hashing mode the goal is to find and exploit the weakest of all keys. We use the fact that the key 0 in IDEA is extremely weak, actually rendering the whole encryption process a T-function [23], already known as dangerous for building a hash function [34]. While weak-keys are already known to be dangerous for block cipher-based hash functions, our method use a novel and non-trivial almost half-involution property for IDEA. Even strengthened versions of the cipher with any number of rounds can be attacked with about the same complexities. This work is one more example that one has to be very careful when hashing with a block cipher that presents any weakness when the key is known or controlled by the attacker. In particular, one should strictly avoid the use of a block cipher for which weak-keys exist, even if only a single weak-key is known.

\section{The IDEA Block Cipher}

The International Data Encryption Algorithm (IDEA) is a 64-bit block cipher handling 128-bit keys and designed by Lai and Massey 26 39] in 1990. While its use is reducing over the recent years, it remains deployed in practice and has not been broken yet despite its advanced age. It has a very simple design, performing 8.5 rounds composed of only 16-bit wide XOR, additions and multiplications. More precisely, one round is composed of three layers: first the key addition layer (denoted KA), a multiplication-addition layer (denoted MA) and a middle words switching layer (denoted $\mathrm{S}$ ). For the eighth round, the switching is omitted.

Let $X^{i}$ represent the 64-bit internal state of IDEA before application of the $i$-th round and we can view it as four 16-bit subwords $X^{i}=\left(X_{1}^{i}, X_{2}^{i}, X_{3}^{i}, X_{4}^{i}\right)$, with $1 \leq i \leq 9$. Also, $Y^{i}=\left(Y_{1}^{i}, Y_{2}^{i}, Y_{3}^{i}, Y_{4}^{i}\right)$ will stand for the intermediate internal state value of IDEA during the $i$-th round, right between the KA and the MA layers. We denote by $\oplus$ the bitwise XOR operation, by $\boxplus$ the addition modulo $2^{16}$ and by $\odot$ the multiplication modulo $2^{16}+1$, where the value 0 is considered as $2^{16}$ and vice-versa. Finally, $Z^{i}=\left(Z_{1}^{i}, Z_{2}^{i}, Z_{3}^{i}, Z_{4}^{i}, Z_{5}^{i}, Z_{6}^{i}\right)$ represents the six 16 -bit subkeys used during the $i$-th round (only the first four subkeys for the last half round).

The KA layer simply incorporates four subkeys:

$$
Y_{1}^{i}=X_{1}^{i} \odot Z_{1}^{i}, \quad Y_{2}^{i}=X_{2}^{i} \boxplus Z_{2}^{i}, \quad Y_{3}^{i}=X_{3}^{i} \boxplus Z_{3}^{i}, \quad Y_{4}^{i}=X_{4}^{i} \odot Z_{4}^{i} .
$$

The MA layer first computes $B=Z_{6}^{i} \odot\left(\left(Y_{2}^{i} \oplus Y_{4}^{i}\right) \boxplus\left(Z_{5}^{i} \odot\left(Y_{1}^{i} \oplus Y_{3}^{i}\right)\right)\right)$ and $A=B \boxplus\left(Z_{5}^{i} \odot\left(Y_{1}^{i} \oplus Y_{3}^{i}\right)\right)$. Then, after application of the $\mathrm{S}$ layer we have:

$$
X_{1}^{i+1}=Y_{1}^{i} \oplus B, \quad X_{2}^{i+1}=Y_{3}^{i} \oplus B, \quad X_{3}^{i+1}=Y_{2}^{i} \oplus A, \quad X_{4}^{i+1}=Y_{4}^{i} \oplus A .
$$


All the subkeys are simply determined by choosing consecutive bits in the 128-bit master key according to some selection table (we refer to the IDEA specifications). Finally, ciphering the plaintext $P$ with IDEA to obtain the ciphertext $C$ is defined as: $C=\mathrm{KA} \circ \mathrm{S} \circ\{\mathrm{S} \circ \mathrm{MA} \circ \mathrm{KA}\}^{8}(P)$.

Currently, the best cryptanalysis work published on IDEA [5] can reach 7.5 rounds with $2^{63}$ data (half the codebook) and $2^{114}$ computations. Concerning weak-keys, the current biggest weak-key class contains $2^{64}$ elements and has been published in [6].

\section{Hashing with a Double-Length Key Block Cipher}

We will study the security of the various block cipher-based constructions that can use IDEA as the internal primitive. Therefore, we only consider the ones that use a double-key block cipher. More precisely, we denote $C=E_{K}(P)$ the process of ciphering the 64-bit plaintext $P$ with IDEA using the 128-bit key $K$.

\subsection{Simple-Length Compression Function}

A simple-length compression function construction with IDEA will provide a 64bit output $C V_{i+1}$.

Davies-Meyer is the most usual simple-length mode [1] and it handles 128-bit message blocks: $C V_{i+1}=E_{M}\left(C V_{i}\right) \oplus C V_{i}$. Most standardized hash functions are actually implementing this mode, with an ad-hoc internal block cipher. While some weaknesses such as fixed-points are known, its security in terms of preimage and collision resistance have been studied and proved in the ideal cipher model [7]. Namely, we should expect at least $2^{32}$ and $2^{64}$ computations respectively to generate a (semi)-free-start collision or preimage for the compression function. Note that Miyaguchi-Preneel and Matyas-Meyer-Oseas simple-block length modes [1] are not considered in this article since they require the internal primitive to have the same block and key size, which is not the case for IDEA.

\subsection{Double-Length Compression Function}

A more interesting design strategy with IDEA would be to define double-block length constructions, in order to get 128-bit output, represented by two 64-bit words $C V 1_{i}$ and $C V 2_{i}$. This problem has already been studied a lot and remains a very active research domain, even when the internal primitive is a double-key block cipher.

Abreast-DM and Tandem-DM will of course be considered in this article since they both have been especially designed for IDEA [27/39]. Tandem-DM handles a 64-bit message block $M$. 
We define $W=E_{C V 1_{i} \| M}\left(C V 2_{i}\right)$ and then we have

$$
\begin{aligned}
& C V 1_{i+1}=E_{M \| W}\left(C V 1_{i}\right) \oplus C V 1_{i}, \\
& C V 2_{i+1}=W \oplus C V 2_{i} .
\end{aligned}
$$

Abreast-DM also handles a 64-bit message block $M$ :

$$
\begin{aligned}
& C V 1_{i+1}=E_{M \| C V 2_{i}}\left(\overline{C V}_{i}\right) \oplus C V 1_{i}, \\
& C V 2_{i+1}=E_{C V 1_{i} \| M}\left(C V 2_{i}\right) \oplus C V 2_{i},
\end{aligned}
$$

where $\bar{X}$ stands for the bitwise complement of $X$.

Hirose proposed a construction that contains two independent block cipher instances [19], later improved to only a single instance [20] by using a constant $c$ to simulate the two independent ciphers:

$$
\begin{aligned}
& C V 1_{i+1}=E_{C V 2_{i} \| M}\left(C V 1_{i}\right) \oplus C V 1_{i}, \\
& C V 2_{i+1}=E_{C V 2_{i} \| M}\left(C V 1_{i} \oplus c\right) \oplus C V 1_{i} \oplus c .
\end{aligned}
$$

Peyrin et al. described in 35] a compression function (denoted Peyrin et al.(II)) that utilizes 5 calls to independent $3 n$-to- $n$-bit compression functions, advising to be instantiated with double-key internal block ciphers such as AES256 or IDEA. It handles two 64-bit message blocks $M 1$ and $M 2$ :

$$
\begin{aligned}
& C V 1_{i+1}=f_{1}\left(C V 1_{i}, C V 2_{i}, M 1\right) \oplus f_{2}\left(C V 1_{i}, C V 2_{i}, M 2\right) \oplus f_{3}\left(C V 1_{i}, M 1, M 2\right), \\
& C V 2_{i+1}=f_{3}\left(C V 1_{i}, M 1, M 2\right) \oplus f_{4}\left(C V 1_{i}, C V 2_{i}, M 1\right) \oplus f_{5}\left(C V 2_{i}, M 1, M 2\right),
\end{aligned}
$$

where the functions $f_{i}$ can be build for example by using the IDEA block cipher into a Davies-Meyer mode and we can simulate their independency by XORing distinct constants to the plaintext inputs, as it is done in [20]: $f_{i}(U, V, W)=$ $E_{U|| V}(W \oplus i) \oplus W$ (note that XORing the constants on the key input would be avoided in practice because it would lead to very frequent rekeying and therefore reduce the overall performance of the hash function). Since no real candidate was proposed by the authors, all possible position permutations of the three $f_{i}$ inputs will be considered. Note that when cryptanalysing this scheme, we will attack the functions $f_{i}$ independently. Thus, we will not use any weakness coming from potential dependencies between the functions $f_{i}$ (apart of course that all 5 functions are based on IDEA).

MJH-Double is a rate 1 double-block length compression function recently published by Lee and Stam [29]. It uses a double-key block cipher and handles two 64-bit message blocks $M 1$ and $M 2$ :

$$
\begin{aligned}
& C V 1_{i+1}=E_{M 2 \| C V 2_{i}}\left(C V 1_{i} \oplus M 1\right) \oplus C V 1_{i} \oplus M 1, \\
& C V 2_{i+1}=g \cdot\left(E_{M 2 \| C V 2_{i}}\left(f\left(C V 1_{i} \oplus M 1\right)\right) \oplus f\left(C V 1_{i} \oplus M 1\right)\right) \oplus C V 1_{i},
\end{aligned}
$$

where $f$ is an involution with no fixed point and $g \neq 0,1$ is a constant. 
For all these double-block length proposals, the conjectured security is $2^{64}$ and $2^{128}$ computations respectively to generate a (semi)-free-start collision or preimage for the compression function or hash function.

\section{Weak-Keys for IDEA}

Weak-keys for IDEA has already been studied in details 610 18, but what we are looking for is slightly different. Indeed, for block cipher cryptanalysis, since the attacker can not control the key input he looks for the biggest possible class of weak-keys, so as to get the highest possible probability that a weak-key will indeed be chosen. In the case of compression function cryptanalysis, the key input is fully known or even controlled by the attacker. The goal is therefore not to find the biggest possible class of weak-keys, but to find the weakest possible key. As we will show for IDEA, even if only one weak-key is found, its weakness might directly lead to successful attacks on the whole compression or hash function.

\subsection{Analysis of the Internal Functions}

When looking at the internal round function of IDEA, one might wonder what would be a weak-key. In IDEA, the most annoying functions for the cryptanalyst are clearly the multiplications in $\mathbb{Z}_{2^{16}+1}$. Indeed, these operations are strongly non-linear and provide good diffusion between the different bit positions. On the contrary, XOR operations are linear and do not provide any diffusion between the bit positions, while the additions in $\mathbb{Z}_{2^{16}}$ can be easily approximated linearly and the diffusion between the bit positions only happens through the carry. Moreover, $\mathrm{XOR}$ and additions are even weaker in IDEA since no rotations are present, comparing with Addition-Rotation-XOR (ARX) designs. Here the rotation is done through the multiplications in $\mathbb{Z}_{2^{16}+1}$ and our goal is therefore to avoid them.

When adding $(a+b) \bmod 2^{16}$, we can avoid any diffusion by forcing one operand to 0 . When multiplying $(a \odot b)=(a \cdot b) \bmod 2^{16}+1$, the good diffusion will happen especially when $(a \cdot b) \geq 2^{16}+1$. An easy way to avoid this is to fix one of the two operands to 1 . In that case, we have $(a \odot 1)=(a \cdot 1) \bmod 2^{16}+1=$ $a \bmod 2^{16}$. As already remarked in [10], a good choice is also 0 , since

$$
\begin{aligned}
(a \odot 0) \bmod 2^{16} & =\left(\left(a \cdot 2^{16}\right) \bmod \left(2^{16}+1\right)\right) \bmod 2^{16} \\
& =\left(\left(\left(a \cdot 2^{16}+a\right)+\left(2^{16}+1\right)-a\right) \bmod \left(2^{16}+1\right)\right) \bmod 2^{16} \\
& =\left(0+2^{16}+1-a\right) \bmod 2^{16}=1-a \bmod 2^{16} \\
& =2+\left(2^{16}-1-a\right) \bmod 2^{16}=(2+\bar{a}) \bmod 2^{16}
\end{aligned}
$$

and the multiplication is reduced to only a complement and an addition with a constant. 


\subsection{Weak-Keys Classes}

Based on the remark that the operand 0 is very weak for both multiplications and additions, Daemen et al. [10] generated a class of weak-keys. A first obvious candidate is the null key (all bits set to zero), which will force all the subkeys to zero as well. As a consequence, all subkeys additions can be simply removed and all subkeys multiplications can be replaced by a complement (or XOR with Oxffff) and an addition with value 2 . At this point, all the operations in IDEA with null key are either XOR or additions. Therefore, by inserting differences only on the Most Significant Bit (MSB) of the four 16-bit plaintext input words, the attacker is ensured that only the MSB of the four output words will contain a difference. Even better, the mapping from an MSB input difference pattern to an MSB output difference pattern is completely deterministic (is it linear since on the MSB no carry is propagated). Such a property is largely sufficient to consider the null key as weak. This reasoning can be generalized by observing that the attacker does not necessarily need all subkeys to be null, but only the ones that are multiplied to an internal word which contains a MSB difference. Since the MSB differential paths are quite sparse, many of the null constraints on the subkeys are relaxed and one finally gets $2^{35}$ weak-keys.

\subsection{The Null Weak-Key}

We show that the null key is particularly weak for hash function utilization. Even if other keys belong to a weak-key class, they do not present the same special properties as the null key.

Almost Half-Involution. When using the null key, we remark that all subkeys will be null as well. Then, all rounds layers will be the same and we write $\mathrm{KA}_{0}$ and $\mathrm{MA}_{0}$ the KA and MA layers with null subkeys. A nice practical feature of IDEA is that the decryption is done using the very same algorithm as encryption, but with different subkeys. The decryption subkeys for the MA layer are the same as the encryption ones since the MA layer is an involution (i.e. $\mathrm{MA}=\mathrm{MA}^{-1}$ ). The decryption subkeys for the KA layer are the respective multiplicative and additive inverses of the encryption subkeys. However, note that a null subkey is both its own multiplicative and additive inverse and the KA layer becomes an involution as well (i.e. $\mathrm{KA}_{0}=\mathrm{KA}_{0}^{-1}$ ). To summarize, using the null key, we are ensured that $\mathrm{KA}_{0}=\mathrm{KA}_{0}^{-1}$ and $\mathrm{MA}_{0}=\mathrm{MA}_{0}^{-1}$. Note that we trivially have $\mathrm{S}=\mathrm{S}^{-1}$.

Now, since the KA layer and $\mathrm{S}$ layer commute, IDEA with null key can be rewritten as

$$
\begin{aligned}
C & =\mathrm{KA}_{0} \circ \mathrm{S} \circ\left\{\mathrm{S} \circ \mathrm{MA}_{0} \circ \mathrm{KA}_{0}\right\}^{8}(P) \\
& =\mathrm{KA}_{0} \circ \mathrm{S} \circ\left\{\mathrm{S} \circ \mathrm{MA}_{0} \circ \mathrm{KA}_{0}\right\}^{3} \circ \mathrm{S} \circ \mathrm{MA}_{0} \circ \mathrm{KA}_{0} \circ\left\{\mathrm{S} \circ \mathrm{MA}_{0} \circ \mathrm{KA}_{0}\right\}^{4}(P) \\
& =\underbrace{\mathrm{KA}_{0} \circ \mathrm{MA}_{0} \circ\left\{\mathrm{S} \circ \mathrm{KA}_{0} \circ \mathrm{MA}_{0}\right\}^{3}}_{\sigma^{-1}} \circ \underbrace{\mathrm{KA}_{0} \circ \mathrm{S}}_{\theta} \circ \underbrace{\left\{\mathrm{MA}_{0} \circ \mathrm{KA}_{0} \circ \mathrm{S}\right\}^{3} \circ \mathrm{MA}_{0} \circ \mathrm{KA}_{0}}_{\sigma}(P)
\end{aligned}
$$


which eventually gives $C=\sigma^{-1} \circ \theta \circ \sigma(P)$. One can check that since $\mathrm{KA}_{0}, \mathrm{MA}_{0}$ and $\mathrm{S}$ are involutions, the operation denoted by $\sigma^{-1}$ is indeed the inverse of the one denoted by $\sigma$. Thus, using the notation

$$
P \stackrel{\sigma^{-1}}{\longrightarrow} U \stackrel{\theta}{\longrightarrow} V \stackrel{\sigma}{\longrightarrow} C
$$

where $U$ and $V$ are internal state values, we have

$$
P \stackrel{\sigma}{\longleftarrow} U \stackrel{\theta}{\longrightarrow} V \stackrel{\sigma}{\longrightarrow} C .
$$

We will use this almost half-involution property in Section [6 to find free-start collisions and even hash function collisions for some IDEA-based constructions.

T-function. When using the null key, we have already described that all operations remaining are either XOR or additions. These operations are triangular functions 23. (or T-functions) in the sense that any output bit at position $i$ only depends on the input bits located at a position $i$ or lower. A composition of T-functions is itself a T-function, therefore the whole permutation defined by IDEA with the null key is a T-function. As shown in [34, this property might be very dangerous in a hash function design. We will explain in Section 7 how to exploit this weakness and compute preimages by guessing the input words bit layer by bit layer.

\section{Simple Collision Attacks}

As shown by Daemen et al. 10, when using the null key for the encryption process of IDEA, differences inserted uniquely on the MSB of the four 16-bit input plaintext words will lead to differences on the MSB of the four 16-bit output ciphertext words. Moreover, since this difference mapping is linear (the difference on the carry is not propagated further than the MSB), all possible differential characteristics have a differential probability 1 . For example, we denote by $\delta_{M S B}=0 \times 8000$ the 16-bit word with difference only on the MSB and by $\Delta_{M S B}=\left(\delta_{M S B}, \delta_{M S B}, \delta_{M S B}, \delta_{M S B}\right)$ the 64-bit difference composed of 4 words with difference $\delta_{M S B}$. Then, $\Delta_{M S B}$ propagates to itself with probability 1 through one round of IDEA, or through its last half-round. Therefore, we have with probability 1

$$
\Delta_{M S B} \stackrel{{ }^{\mathrm{IDEA}_{K=0}} \longrightarrow}{\longrightarrow} \Delta_{M S B} .
$$

Note that instead of using $\delta_{M S B}$ only, one can generalize the input difference space and obtain other very good differential paths for the encryption of IDEA with the null key. However, we omit this generalization here since the methods described in later sections already provide much better attacks. 
Davies-Meyer. Finding a free-start collision on Davies-Meyer mode instantiated with IDEA is very easy. Since the difference $\Delta_{M S B}$ is mapped to itself through the IDEA encryption process with the null key, the attacker only has to pick $M=0$. Then, any value of $C V$ with difference $\Delta_{M S B}$ applied to it will lead to a collision with probability 1 . We give in the full version of the article examples of such a free-start collision.

Hirose. The same method as for Davies-Meyer mode can be applied to the Hirose mode in order to find free-start collisions. The attacker fixes $C V 2=0$ and $M=$ 0 so as to force the null key to both encryptions. Then, any value of $C V 1$ with a difference $\Delta_{M S B}$ applied to it will lead to a collision with probability 1 , since $\Delta_{M S B}$ will appear on the plaintext input of both encryptions with the null key. We give in the full version of the article examples of such a free-start collision.

Abreast-DM. This technique seems impossible to apply to the Abreast-DM mode since forcing a difference $\Delta_{M S B}$ on any of the two encryptions plaintext input will imply a difference inserted in the key input of the other encryption block. Therefore, one cannot use $\Delta_{M S B}$ difference on plaintext input with null key in both encryption blocks. Even if the attacker tries to attack only one encryption block with this method, the other block will not be controlled and he will have to deal with random differences on its output. These random differences cannot be dealt with some birthday technique because fixing all inputs of one encryption block will fix all inputs of the other one as well.

Tandem-DM. This technique seems impossible to apply to the Tandem-DM mode for the exact same reasons as for Abreast-DM.

Peyrin et al.(II). We have to separate in two groups the possible instances of this construction, obtained by permuting the position of the three inputs of each internal function $f_{i}$. If all compression function inputs $C V 1, C V 2, M 1$ and $M 2$ appear in at least one of the IDEA key inputs of any $f_{i}$ internal function, then the attack will not apply. Indeed, since all inputs will be involved at least one time, the attacker will necessarily have to insert a difference in at least one IDEA key input and he will not be able to use the differential path with probability 1 . Note that these instances would be avoided in practice because they would lead to more frequent re-keying and therefore reduce the overall performance of the hash function. If this condition is not met, then we can apply the following free-start collision attack. Let $X \in\{C V 1, C V 2, M 1, M 2\}$ denote the input that is missing in all the IDEA key inputs of the compression function. The attacker simply fixes the difference $\Delta_{M S B}$ on $X$ (one can give any value to $X$ ) and all other inputs are set to 0 in order to get the null key in every internal IDEA. The attacker ends up with several Davies-Meyer in parallel, with either no difference at all or with null key and $\Delta_{M S B}$ as plaintext input difference. Thus, he obtains a collision with probability 1. If $X \notin\{C V 1, C V 2\}$, then this attack finds semi-free-start collisions. 
MJH-Double. The MJH-Double mode prevents this simple attack since even if we fix $C V 2=0$ and $M 2=0$ in order to get the null key in both encryptions, it is hard to force the difference $\Delta_{M S B}$ on both their plaintext inputs. Indeed, the $f$ operation will randomize the difference and in order for the attack to run, we would require $\Delta_{M S B} \stackrel{f}{\longrightarrow} \Delta_{M S B}$ which is unlikely to happen.

\section{Improved Collision Attacks}

In this section, using the almost half-involution property with the null key, we will show how to get the same difference on the input and on the output of the IDEA ciphering process with good probability. Then, we will use this weakness to derive our collision attacks, for any number of rounds.

\subsection{Exploiting the Almost Half-Involution}

We have already shown in Section 4 that when the key is null, IDEA encryption process can be rewritten as

$$
P \stackrel{\sigma}{\longleftarrow} U \stackrel{\theta}{\longrightarrow} V \stackrel{\sigma}{\longrightarrow} C
$$

where

$$
\sigma=\left\{\mathrm{MA}_{0} \circ \mathrm{KA}_{0} \circ \mathrm{S}\right\}^{3} \circ \mathrm{MA}_{0} \circ \mathrm{KA}_{0} \quad \text { and } \quad \theta=\mathrm{KA}_{0} \circ \mathrm{S} .
$$

We denote $\Delta U$ the XOR difference between two 64-bit internal state values $U$ and $U^{\prime}$, i.e $\Delta U=U \oplus U^{\prime}$, and $\delta U_{i}$ represents the 16-bit difference on the $i$-th word of $\Delta U$, that is $\Delta U=\left(\delta U_{1}, \delta U_{2}, \delta U_{3}, \delta U_{4}\right)$. Let us consider two random 64-bit internal state values $U$ and $U^{\prime}$ such that $\delta U_{2}=\delta U_{3}$ and we denote this 16-bit difference $\delta_{M}$. For truly random values $U$ and $U^{\prime}$, this condition happens with probability $2^{-16}$. One can check that applying $\theta$ on $U$ and $U^{\prime}$ to obtain $V$ and $V^{\prime}$ respectively will lead to $\delta V_{2}=\delta V_{3}=\delta_{M}$ since layer S only switches the two middle words and layer $\mathrm{KA}_{0}$ has no effect on them (addition of null subkeys).

Let $\delta_{L}$ and $\delta_{R}$ represent the difference on $\delta U_{1}$ and $\delta U_{4}$ respectively, i.e. $\Delta U=$ $\left(\delta_{L}, \delta_{M}, \delta_{M}, \delta_{R}\right)$. Applying function $\theta$ to $U$ and $U^{\prime}$, we would like the same differences to appear on internal state $V$ and $V^{\prime}: \Delta V=\left(\delta_{L}, \delta_{M}, \delta_{M}, \delta_{R}\right)$. The previous condition with probability $2^{-16}$ already ensures the two middle differences being the same $\delta_{M}$. Concerning differences $\delta_{L}$ and $\delta_{R}$, they will both be unaffected by layer $\mathrm{S}$, but they might be modified through layer $\mathrm{KA}_{0}$ that applies a multiplication with a null subkey. Therefore, we need to study the probability that a random difference $\delta$ is mapped to itself through a multiplication by the null subkey. We show in the full version of the article that this probability is equal to $2^{-1.585}$ and finally we have $\operatorname{Pr}\left[\left(\delta_{L}, \delta_{M}, \delta_{M}, \delta_{R}\right) \stackrel{\theta}{\longrightarrow}\left(\delta_{L}, \delta_{M}, \delta_{M}, \delta_{R}\right)\right]=2^{-3.17}$.

At this point, we proved that for randomly chosen internal state values $U$ and $U^{\prime}$, we will observe with probability $2^{-19.17}$ the same difference on $U$ and $V$, i.e. $\Delta U=\Delta V$. 
One can see that computing backward from internal states $U$ to $P$ or forward from $V$ to $C$, the function $\sigma$ is applied. Our final goal is to have the same difference on $P$ and $C$. However, this seems unlikely to happen since $U$ and $V$ have different values, the forward and backward computations of $\sigma$ should be completely unrelated, even with the same input difference. Yet, this reasoning does not take in account the fact that while $U$ and $V$ have distinct values, they are far from being independent: $V=\theta(U)$ with $\theta$ being a very light function. Moreover, we remarked that almost each time that we got the same difference on $P$ and $C$, the same differences were observed as well in all rounds of the forward and backward $\sigma$ computations (the round success probability increasing with the number of rounds already processed). Because all the rounds are not independent and because $U$ and $V$ are strongly related, it is very difficult to compute theoretically the probability of observing the same difference on $P$ and $C$ and we leave this as an open problem. Therefore, we measured it by choosing random values of $U, \delta_{L}, \delta_{M}, \delta_{R}$, computing $V=\theta(U)$, and checking for collisions on the difference of $P$ and $C$. The probability obtained was $2^{-16.26}$ for about $2^{28}$ tests (note that this probability somehow contains the $2^{-3.17}$ probability computed previously, but we can not separate them because the two events are not independent).

To conclude, the probability that two randomly chosen internal state values $U$ and $U^{\prime}$ give the same difference on $P$ and $C$ is equal to $2^{-16-16.26}=2^{-32.26}$ (instead of $2^{-64}$ expected for a random function). In other words, using the birthday paradox, one can find such a pair with about $2^{16.13}$ computations.

Interestingly, we have observed that most of the pairs fulfilling the differential path for the full IDEA will also be valid for a strengthened version of the cipher with any number of additional rounds. Since the subkeys are always null, strengthening the cipher would mean that $\sigma=\left\{\mathrm{MA}_{0} \circ \mathrm{KA}_{0} \circ \mathrm{S}\right\}^{t} \circ \mathrm{MA}_{0} \circ \mathrm{KA}_{0}$ for any $t>3$. We checked that the probability that two randomly chosen internal state values $U$ and $U^{\prime}$ give the same difference on $P$ and $C$ tends to $2^{-32.54}$ when $t$ tends to infinite. Thus, similarily to the method presented in the previous section, the attacks using this almost half-involution property will work for any number of rounds.

\subsection{Improving Collision Attacks}

Davies-Meyer. A first obvious application of having the same difference in $P$ and $C$ is collision search on Davies-Mayer mode, where the feed-forward will cancel the two differences in the output. The attack finds collisions for the whole hash function and the procedure is very simple: we start from the IV and add random differences in the first message block $M_{0}$. This will cause random differences in the the first chaining variable $C V_{1}$. For the second message block $M_{1}$, we will set all its bits $0\left(M_{1}=0\right)$, forcing the internal IDEA computation to use the null key. Since we estimated in the previous section that with the null key a random pair of inputs has a probability $2^{-32.26}$ to give the same input/output difference, one can use the birthday paradox to generate a collision on $C V_{2}$ with only $2^{16.13}$ distinct message blocks $M_{0}$. We give in the full version of the article examples of hash collisions for the Davies-Meyer mode. Note that finding 
semi-free-start collisions with this technique is impossible since we would have to insert differences in the message input, which forbids the use of the null key in the internal cipher.

Hirose. We already showed how to find free-start collisions for the Hirose mode. However, finding semi-free-start collisions with this technique is impossible since we would have to insert differences in the message input, which forbids the use of the null key in the internal cipher. Also, concerning hash collisions, it seems hard as well because forcing the null key during iteration $i$ requires us to obtain a chaining variable $C V 2_{i-1}=0$ during the previous iteration. This half-preimage already costs the same complexity as a generic collision search on the entire compression function.

Abreast-DM. One can derive a free-start collision attack for the Abreast-DM compression function using this technique. The attacker first fixes $C V 1=0$ and $M=0$. Then, he builds a set of $2^{48.13}$ distinct values $C V 2$ and checks if a pair of this set leads to a collision. The probability that a pair leads to a collision on the first (top) branch is $2^{-32.26}$ (since the internal cipher on this part has the null key), and $2^{-64}$ on the other half. Overall, using the birthday paradox on the set of $2^{48.13}$ values $C V 2$ is sufficient to have a good chance to obtain a collision. Note that finding a semi-free-start collision for the compression function or a collision for the hash function seems impossible with this method, for the same reasons as the Hirose mode.

Tandem-DM. The situation of Tandem-DM is absolutely identical to the Abreast-DM one: one can find free-start collisions for compression function using this technique. The attacker first fixes $C V 1=0$ and $M=0$. Then, he builds a set of $2^{48.13}$ distinct values $C V 2$ and checks if a pair of this set leads to a collision. The probability that a pair leads to a collision on the first (top) branch is $2^{-32.26}$ (since the internal cipher on this part has the null key), and $2^{-64}$ on the other half. Overall, using the birthday paradox on the set of $2^{48.13}$ values $C V 2$ is sufficient to have a good chance to obtain a collision. Again, finding a semi-free-start collision for the compression function or a collision for the hash function seems impossible with this method, for the same reasons as the Hirose mode.

Peyrin et al.(II). We showed in previous section how to find (semi)-free-start collisions with probability 1 for a certain subset of Peyrin et al.(II) constructions, but here we provide attacks on a bigger subset. If all compression function inputs $C V 1, C V 2, M 1$ and $M 2$ appear in at least one of the IDEA key inputs of $f_{1}$, $f_{2}, f_{3}$ (left side) and in at least one of the IDEA key inputs of $f_{3}, f_{4}, f_{5}$ (right side), then the attack will not apply. Indeed, for both left side and right side of the compression function, the attacker will necessarily have to insert a difference in at least one key input (since all inputs will be involved) and he will not be able to use the null key completely. Note that these instances would be avoided in practice because they would lead to more frequent rekeying and therefore 
reduce the overall performance of the hash function. However, if this condition is not met, then we can apply the following free-start collision attack. Let $X \in$ $\{C V 1, C V 2, M 1, M 2\}$ denote the input that is missing in all the IDEA key inputs of $f_{1}, f_{2}, f_{3}$ (wlog the reasoning is the same with $f_{3}, f_{4}, f_{5}$ ). The attacker first fixes all inputs but $X$ to 0 in order to get the null key in every internal IDEA on the left side. Then he chooses $2^{48.13}$ random values for $X$ and checks among them if any pair collides on the whole compression function output. Since he has a probability $2^{-32.26}$ to get a collision on the left side and $2^{-64}$ on the right side, using a birthday search the attacker finds a solution with complexity $2^{48.13}$. Again, if $X \notin\{C V 1, C V 2\}$, then this attack finds semi-free-start collisions. However, finding a collision for the hash function seems impossible with this method, because at least one of the chaining variable inputs $C V 1$ and $C V 2$ will be present as key input for one of the IDEA internal emcryption. Setting this word to 0 is equivalent to a half-preimage that already costs the same complexity as a generic collision search on the entire hash function.

MJH-Double. One can derive a semi-free-start collision attack on the MJHDouble compression function instantiated with IDEA. The attacker first fixes $C V 2=$ 0 and $M 2=0$ and this will force the null key in both encryptions. Now he chooses a random value for $C V 1$ (note that actually this value could be fixed by the challenger) and builds a set of $2^{32.26}$ values $M 1$. In this configuration, it is easy to see that one will have random differences on the plaintext inputs to both encryptions. Since the null key is used for both, we have a probability $2^{-64.52}$ that a pair of $M 1$ leads to a collision after the feed-forward of both encryptions (on the output of the bottom block and just before the application of $g$ on the top block). Therefore, with a birthday technique, one can find such a pair with only $2^{32.26}$ computations. Note that while this pair will directly lead to a collision on the bottom $C V 1$ output, the difference on $M 1$ is injected two times before computing the top $C V 2$ output. Two times of the same difference will cancel themselves and we eventually get a full semifree-start collision. Note that it seems hard to extend this attack to a hash collision since the attacker would require to force the incoming chaining variable $C V 2$ to be equal to 0 and this half-preimage already costs the same complexity as a generic collision search on the entire hash.

\section{Preimage Attacks}

Due to space limitations, all results regarding preimage attacks are given in the full version of the article.

\section{Results and Implementations}

We depict in Table 1 our collision results for the block cipher to compression function modes considered in this article when instantiated with IDEA. We implemented all attacks of reasonable complexities and provide in the full version of the article the collision/preimage examples obtained. 
Table 1. Summary of collision results for block cipher to compression function modes when instantiated with IDEA (we did not include MDC-2 as it does not provide ideal collision resistance). The results for Peyrin et al.(II) construction, marked with a *, depend on the instance considered (see relevant parts of Sections 5 and 6 for more details).

\begin{tabular}{|c||c||c|c||c|}
\hline \multirow{2}{*}{ Mode } & \multirow{2}{*}{\begin{tabular}{c} 
hash \\
output \\
\cline { 5 - 6 }
\end{tabular}} & $\begin{array}{c}\text { free-start } \\
\text { size }\end{array}$ & $\begin{array}{c}\text { semi-free-start } \\
\text { collision attack }\end{array}$ & $\begin{array}{c}\text { collision } \\
\text { collision attack }\end{array}$ \\
\hline \hline Davies-Meyer [1] & 64 & $2^{1}$ & & $2^{16.13}$ \\
\hline \hline Hirose [19[20] & 128 & $2^{1}$ & & \\
\hline Abreast-DM [27]39] & 128 & $2^{48.13}$ & & \\
\hline Tandem-DM [27]39] & 128 & $2^{48.13}$ & & \\
\hline Peyrin et al.(II) [35] & 128 & $2^{1} / 2^{48.13 \star}$ & $2^{1} / 2^{48.13 \star}$ & \\
\hline MJH-Double [29] & 128 & $2^{32.26}$ & $2^{32.26}$ & \\
\hline
\end{tabular}

\section{Conclusion}

In this article, we showed collision and preimage attacks for several single and double-length block cipher based compression function constructions when instantiated with the block cipher IDEA. Namely, we analyzed all known double-key schemes such as Davies-Meyer, Hirose, Abreast-DM, Tandem-DM, Peyrin et al. (II) and MJH-Double. While most of these constructions are conjectured or proved to be secure in the ideal cipher model, we showed that their security is very weak when instantiated with the block cipher IDEA, which remains considered as secure in the secret key model. In particular, we answer in the negative for the 20-year-old standing open question concerning the security of the Abreast-DM and TandemDM instantiated with IDEA. All our practical attacks have been implemented and they can work even for any number of IDEA rounds. Our results indicate that one has to be very careful when hashing with a block cipher that presents any weakness when the key is known or controlled by the attacker. Also, since we extensively use the presence of weak-keys for IDEA, as a future work it would be interesting to look at the security of hash functions based on block ciphers for which some key sets are known to be weaker than others.

Acknowledgments. The authors would like to thank the anonymous referees for their helpful comments.

\section{References}

1. Menezes, A., van Oorschot, P., Vanstone, S.: CRC-Handbook of Applied Cryptography. CRC Press (1996)

2. Ayaz, E.S., Selçuk, A.A.: Improved DST Cryptanalysis of IDEA. In: Biham, E., Youssef, A.M. (eds.) SAC 2006. LNCS, vol. 4356, pp. 1-14. Springer, Heidelberg (2007) 
3. Biham, E., Dunkelman, O., Keller, N.: New Cryptanalytic Results on IDEA. In: Lai and Chen [25], pp. 412-427

4. Biham, E., Dunkelman, O., Keller, N.: A New Attack on 6-Round IDEA. In: Biryukov, A. (ed.) FSE 2007. LNCS, vol. 4593, pp. 211-224. Springer, Heidelberg (2007)

5. Biham, E., Dunkelman, O., Keller, N., Shamir, A.: New Data-Efficient Attacks on Reduced-Round IDEA. Cryptology ePrint Archive, Report 2011/417 (2011)

6. Biryukov, A., Nakahara Jr., J., Preneel, B., Vandewalle, J.: New Weak-Key Classes of IDEA. In: Deng, R.H., Qing, S., Bao, F., Zhou, J. (eds.) ICICS 2002. LNCS, vol. 2513, pp. 315-326. Springer, Heidelberg (2002)

7. Black, J.A., Rogaway, P., Shrimpton, T.: Black-Box Analysis of the Block-CipherBased Hash-Function Constructions from PGV. In: Yung, M. (ed.) CRYPTO 2002. LNCS, vol. 2442, pp. 320-335. Springer, Heidelberg (2002)

8. Brassard, G. (ed.): CRYPTO 1989. LNCS, vol. 435. Springer, Heidelberg (1990)

9. Chang, D.: Near-Collision Attack and Collision-Attack on Double Block Length Compression Functions based on the Block Cipher IDEA. Cryptology ePrint Archive, Report 2006/478 (2006), http://eprint.iacr.org/

10. Daemen, J., Govaerts, R., Vandewalle, J.: Weak Keys for IDEA. In: Stinson [37], pp. $224-231$

11. Daemen, J., Rijmen, V.: The Design of Rijndael: AES - The Advanced Encryption Standard. Springer (2002)

12. Damgård, I.: A Design Principle for Hash Functions. In: Brassard [8], pp. 416-427

13. Demirci, H., Selçuk, A.A., Türe, E.: A New Meet-in-the-Middle Attack on the IDEA Block Cipher. In: Matsui and Zuccherato [32], pp. 117-129

14. den Boer, B., Bosselaers, A.: Collisions for the Compression Function of MD-5. In: Helleseth, T. (ed.) EUROCRYPT 1993. LNCS, vol. 765, pp. 293-304. Springer, Heidelberg (1994)

15. Dobbertin, H.: Cryptanalysis of MD5 compress. Presented at the Rump Session of EUROCRYPT 1996 (1996)

16. Fleischmann, E., Gorski, M., Lucks, S.: On the Security of Tandem-DM. In: Dunkelman, O. (ed.) FSE 2009. LNCS, vol. 5665, pp. 84-103. Springer, Heidelberg (2009)

17. Fleischmann, E., Gorski, M., Lucks, S.: Security of Cyclic Double Block Length Hash Functions including Abreast-DM. Cryptology ePrint Archive, Report 2009/261 (2009), http://eprint.iacr.org/

18. Hawkes, P.: Differential-Linear Weak Key Classes of IDEA. In: Nyberg, K. (ed.) EUROCRYPT 1998. LNCS, vol. 1403, pp. 112-126. Springer, Heidelberg (1998)

19. Hirose, S.: Provably Secure Double-Block-Length Hash Functions in a Black-Box Model. In: Park, C., Chee, S. (eds.) ICISC 2004. LNCS, vol. 3506, pp. 330-342. Springer, Heidelberg (2005)

20. Hirose, S.: Some Plausible Constructions of Double-Block-Length Hash Functions. In: Robshaw, M. (ed.) FSE 2006. LNCS, vol. 4047, pp. 210-225. Springer, Heidelberg (2006)

21. Kelsey, J., Schneier, B., Wagner, D.: Key-Schedule Cryptanalysis of IDEA, G-DES, GOST, SAFER, and Triple-DES. In: Koblitz, N. (ed.) CRYPTO 1996. LNCS, vol. 1109, pp. 237-251. Springer, Heidelberg (1996)

22. Khovratovich, D., Leurent, G., Rechberger, C.: Narrow-Bicliques: Cryptanalysis of Full IDEA. In: Pointcheval, D., Johansson, T. (eds.) EUROCRYPT 2012. LNCS, vol. 7237, pp. 392-410. Springer, Heidelberg (2012)

23. Klimov, A., Shamir, A.: Cryptographic Applications of T-Functions. In: Matsui and Zuccherato [32], pp. 248-261 
24. Knudsen, L.R., Rijmen, V.: Known-Key Distinguishers for Some Block Ciphers. In: Kurosawa, K. (ed.) ASIACRYPT 2007. LNCS, vol. 4833, pp. 315-324. Springer, Heidelberg (2007)

25. Lai, X., Chen, K. (eds.): ASIACRYPT 2006. LNCS, vol. 4284. Springer, Heidelberg (2006)

26. Lai, X., Massey, J.L.: A Proposal for a New Block Encryption Standard. In: Damgård, I.B. (ed.) EUROCRYPT 1990. LNCS, vol. 473, pp. 389-404. Springer, Heidelberg (1991)

27. Lai, X., Massey, J.L.: Hash Functions Based on Block Ciphers. In: Rueppel, R.A. (ed.) EUROCRYPT 1992. LNCS, vol. 658, pp. 55-70. Springer, Heidelberg (1993)

28. Lee, J., Kwon, D.: The Security of Abreast-DM in the Ideal Cipher Model. Cryptology ePrint Archive, Report 2009/225 (2009), http://eprint.iacr.org/

29. Lee, J., Stam, M.: MJH: A Faster Alternative to MDC-2. In: Kiayias, A. (ed.) CT-RSA 2011. LNCS, vol. 6558, pp. 213-236. Springer, Heidelberg (2011)

30. Lee, J., Stam, M., Steinberger, J.: The Collision Security of Tandem-DM in the Ideal Cipher Model. In: Rogaway, P. (ed.) CRYPTO 2011. LNCS, vol. 6841, pp. 561-577. Springer, Heidelberg (2011)

31. Luby, M., Rackoff, C.: How to Construct Pseudorandom Permutations from Pseudorandom Functions. SIAM J. Comput. 17(2), 373-386 (1988)

32. Matsui, M., Zuccherato, R.J. (eds.): SAC 2003. LNCS, vol. 3006. Springer, Heidelberg (2004)

33. Merkle, R.C.: One Way Hash Functions and DES. In: Brassard [8], pp. 428-446

34. Muller, F., Peyrin, T.: Cryptanalysis of T-Function-Based Hash Functions. In: Rhee, M.S., Lee, B. (eds.) ICISC 2006. LNCS, vol. 4296, pp. 267-285. Springer, Heidelberg (2006)

35. Peyrin, T., Gilbert, H., Muller, F., Robshaw, M.J.B.: Combining Compression Functions and Block Cipher-Based Hash Functions. In: Lai and Chen [25], pp. 315-331

36. Preneel, B., Govaerts, R., Vandewalle, J.: Hash Functions Based on Block Ciphers: A Synthetic Approach. In: Stinson [37], pp. 368-378

37. Stinson, D.R. (ed.): CRYPTO 1993. LNCS, vol. 773. Springer, Heidelberg (1994)

38. Wang, X., Yu, H.: How to Break MD5 and Other Hash Functions. In: Cramer, R. (ed.) EUROCRYPT 2005. LNCS, vol. 3494, pp. 19-35. Springer, Heidelberg (2005)

39. Lai, X.: On the Design and Security of Block Ciphers. Hartung-Gorre Verlag, Kon$\operatorname{stanz}(1992)$ 